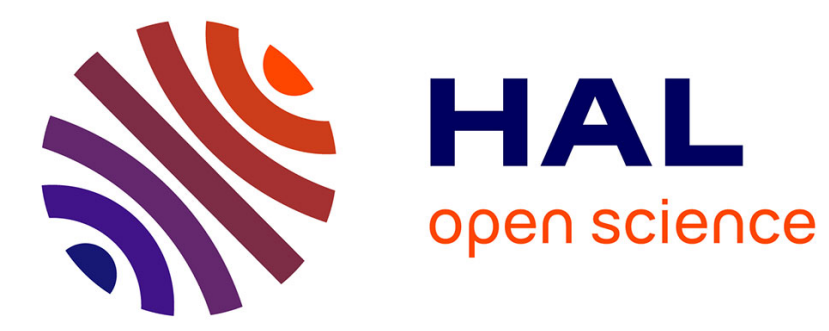

\title{
Prévision du bruit de raie émis par un rotor : application à l'aéronautique
}

\author{
S. Léwy
}

\section{To cite this version:}

S. Léwy. Prévision du bruit de raie émis par un rotor: application à l'aéronautique. Journal de Physique IV Proceedings, 1994, 04 (C5), pp.C5-55-C5-65. 10.1051/jp4:1994506 jpa-00252768

\section{HAL Id: jpa-00252768 https://hal.science/jpa-00252768}

Submitted on 1 Jan 1994

HAL is a multi-disciplinary open access archive for the deposit and dissemination of scientific research documents, whether they are published or not. The documents may come from teaching and research institutions in France or abroad, or from public or private research centers.
L'archive ouverte pluridisciplinaire HAL, est destinée au dépôt et à la diffusion de documents scientifiques de niveau recherche, publiés ou non, émanant des établissements d'enseignement et de recherche français ou étrangers, des laboratoires publics ou privés. 


\title{
Prévision du bruit de raie émis par un rotor : application à l'aéronautique
}

\author{
S. LÉWY
}

Office National d'Etudes et de Recherches Aérospatiales, BP. 72, 92322 Châtillon cedex, France

\begin{abstract}
Résumé : La prévision du bruit de raie des rotors, en vue de la réduction de leur émission sonore, est une préoccupation importante dans le domaine industriel et aéronautique. Deux approches fréquentielles sont exposées ; la première s'applique aux rotors libre, la seconde résout l'équation d'onde dans un conduit cylindrique. Il est montré que les termes dominants sont les mêmes dans les deux cas. Cette constatation est particulièrement intéressante pour les soufflantes transsoniques des turboréacteurs car les calculs établis pour les hélices isolées donnent des résultats valables. L'accent est mis sur les différences par rapport aux directivités rayonnées par les hélices subsoniques classiques.
\end{abstract}

\begin{abstract}
Prediction of rotor tone noise is a major issue both in industry and in aeronautics for reducing sound radiation. Two frequency methods are described, the first applies to open rotors and the second solves the wave equation inside a cylindrical duct. It is shown that the main terms are the same in both cases. This is particularly useful for studying supersonic fans of turbofans because computations of isolated propellers are then valid. Emphasis is put on the differences with the directivities radiated by conventional subsonic propellers.
\end{abstract}

\section{INTRODUCTION}

Les rotors constituent une source de bruit industriel qui est intense, en raison des grandes vitesses périphériques, et gênante, parce que le spectre acoustique est dominé par des raies (harmoniques de la fréquence de passage des aubes). L'aéronautique est évidemment fortement intéressée par ce sujet pour les avions à hélices et les hélicoptères. En ce qui concerne les rotors carénés, l'émission sonore des turboréacteurs modernes à double flux et à grand taux de dilution provient essentiellement de la soufflante, située en tête du réacteur ; celle des turbomoteurs d'hélicoptères est également due en grande partie au premier étage de compression.

La réduction du bruit dès le stade des avant-projets implique des prévisions suffisamment précises. Les méthodes semi-empiriques ne s'appliquent qu'à des familles de machines déjà existantes et leur extrapolation à de nouveaux types est toujours très hasardeuse. L'emploi de modèles purement théoriques s'impose donc. L'objectif de cette communication est de dégager leurs possibilités actuelles. Le premier paragraphe expose un calcul classique dans le domaine fréquentiel, pour un rotor libre [1-5]. Le carénage est introduit dans la deuxième partie : un développement de la solution de l'équation d'onde en conduit cylindrique est présenté [6], associé à un calcul de rayonnement en champ libre tel que celui de Tyler et Sofrin [7]. Bien que ce modèle ait été proposé par Goldstein il y a près de vingt ans, il ne semble pas avoir été appliqué jusqu'à présent ; à la connaissance de l'auteur, seul Schulten a suivi récemment une démarche voisine [8]. 
Il est ensuite noté que les termes sources dominants sont les mêmes dans les deux approches mais le rayonnement peut être assez différent selon que le rotor est libre ou caréné. Eversman a cependant montré qu'il existe une grande similitude, aussi bien sur les niveaux sonores que sur les directivités, dans le cas des rotors transsoniques [9]. Ceci présente de l'intérêt pour l'étude de la soufflante des turboréacteurs actuels, qui est souvent transsonique. Les prévisions acoustiques peuvent alors être effectuées à l'aide des programmes établis pour les hélices isolées [10]. Quelques exemples très simples sont présentés : ils permettent de comparer le rayonnement d'un rotor transsonique libre et celui par l'embouchure d'un conduit ; ils dégagent aussi l'évolution des directivités par rapport à une hélice subsonique classique.

\section{FORMULES POUR UN ROTOR NON CARENE}

\subsection{Formule générale}

Soit un repère cartésien Oxyz (fig. 1) en translation uniforme à la vitesse $U$ = a $M$ selon l'axe $x$ (a est la célérité du son et $\mathrm{M}$ le nombre de $\mathrm{Mach})$. La pression sonore en un point repéré par le vecteur $\overrightarrow{\mathrm{x}}(\mathrm{x}, \mathrm{y}, \mathrm{z})$ à l'instant $\mathrm{t}$ est fournie par l'expression suivante [1, chapitre 3] :

$$
\mathrm{p}(\overrightarrow{\mathrm{x}}, \mathrm{t})=\int_{\mathrm{A}} \int_{\tau} \rho_{0} \mathrm{~V}_{\mathrm{n}} \frac{\mathrm{DG}}{\mathrm{D} \tau} \mathrm{d} \tau \mathrm{dA}+\int_{\mathrm{A}} \int_{\tau} \mathrm{f}_{\mathrm{i}} \frac{\partial \mathrm{G}}{\partial \mathrm{y}_{\mathrm{i}}} \mathrm{d} \tau \mathrm{dA}
$$

où le premier terme de droite représente le bruit d'épaisseur et le second le bruit de charge. Le terme quadripolaire n'est pas considéré dans cette communication, bien qu'il ne soit pas forcément négligeable en transsonique. Dans la relation (1), $\rho_{0}$ est la masse volumique de l'air, A désigne les surfaces solides, $\tau$ est le temps retardé (instant d'émission). Les sources sonores sont situées aux points $\vec{y}\left(\mathrm{y}_{1}, \mathrm{y}_{2}, \mathrm{y}_{3}\right.$ ou $\left.x_{0}, y_{0}, z_{0}\right)$ sur $A: V_{n}$ est la composante de la vitesse relative normale aux surfaces ; les $f_{i}(i=1,2,3)$ sont les composantes selon $\mathrm{x}, \mathrm{y}, \mathrm{z}$ des forces par élément de surface appliquées au fluide (avec sommation sur l'indice i dans $\left.f_{i} \partial G / \partial y_{i}\right)$. La fonction de Green $G$ traduit la réponse en $\vec{x}$ à $t$, à une impulsion émise en $\vec{y}$ à $\tau$ :

$$
\mathrm{G}(\overrightarrow{\mathrm{y}}, \tau ; \overrightarrow{\mathrm{x}}, \mathrm{t})=\frac{1}{4 \pi \mathrm{S}_{0}} \delta\left(\mathrm{t}-\tau-\frac{\sigma_{0}}{\mathrm{a}}\right)
$$

où $\delta$ est la distribution de Dirac et $S_{0}$ et $\sigma_{0}$ sont les distances modifiées :

$$
S_{0}=\sqrt{\left(x-x_{0}\right)^{2}+\beta^{2}\left(y-y_{0}\right)^{2}+\beta^{2}\left(z-z_{0}\right)^{2}}, \sigma_{0}=\frac{M\left(x-x_{0}\right)+S_{0}}{\beta^{2}} \text { et } \beta^{2}=1-M^{2}
$$

Il existe essentiellement deux approches pour intégrer la relation (1). La première est exécutée directement dans le domaine temporel, à partir de la solution fournie par Ffowcs Williams et Hawkings [2]. Cette méthode est largement employée pour les hélices et les rotors d'hélicoptères. L'autre manière de calculer, dans le domaine fréquentiel, est exposée dans la suite. Elle est en effet voisine de celle adoptée pour les rotors carénés car elle aboutit à une décomposition du champ sonore en modes spatiaux, qui jouent un rôle fondamental dans la propagation en milieu guidé. Une résolution de ce type est menée notamment par Hanson dans un repère fixe, ce qui correspond au cas du survol d'un récepteur au sol [3]. L'expression (1), quant à elle, est relative à un microphone lié au moteur, c'est-à-dire à l'avion, et représente aussi la configuration d'essais en soufflerie. 


\subsection{Calcul dans Ie domaine fréquentiel}

La transformation de Fourier de (1) fournit directement les composantes spectrales de la pression sonore. Pour un signal de période T, la série de Fourier s'écrit :

$$
p(\vec{x}, t)=\sum_{n=-\infty}^{+\infty} P(\omega, \vec{x}) e^{i \omega t} \text { et } P(\omega, \vec{x})=\frac{1}{T} \int_{-T / 2}^{+T / 2} p(\vec{x}, t) e^{-i \omega t} d t
$$

où $\omega=2 \pi \mathrm{f}=2 \pi \mathrm{n} / \mathrm{T}$ est la pulsation et $\mathrm{f}$ la fréquence (n entier). Comme seule la fonction de Green $\mathrm{G}$ dépend de l'instant de réception $\mathrm{t}$ dans la relation (1), il vient :

$$
P(\omega, \bar{x})=\int_{A} \int_{\tau} \rho_{0} V_{n} \frac{D G_{\omega}}{D \tau} d \tau d A+\int_{A} \int_{\tau} f_{i} \frac{\partial G_{\omega}}{\partial y_{i}} d \tau d A
$$

La transformée de Fourier $\mathrm{G}_{\omega}$ de $\mathrm{G}$ vaut :

$$
\mathrm{G}_{\omega}=\frac{1}{4 \pi \mathrm{TS}_{0}} \mathrm{e}^{-\mathrm{i} \omega\left(\tau+\sigma_{0} / \mathrm{a}\right)}=\frac{1}{4 \pi \mathrm{TS}_{0}} \mathrm{e}^{-\mathrm{iK}\left(\mathrm{at}+\sigma_{0}\right)}
$$

où $\mathrm{K}=\omega / \mathrm{a}$ est le nombre d'ondes. Par suite, la dérivée convective de $\mathrm{G}$ est :

$$
\frac{\mathrm{DG}_{\omega}}{\mathrm{D} \tau}=\alpha \mathrm{G}_{\omega} \text {, avec } \alpha=-\mathrm{i} \frac{\mathrm{aK}}{\beta^{2}}\left[1+\mathrm{M} \frac{\mathrm{x}-\mathrm{x}_{0}}{\mathrm{~S}_{0}}\left[1-\mathrm{i} \frac{\beta^{2}}{\mathrm{KS}_{0}}\right]\right]
$$

et les dérivées spatiales de G s'expriment par :

$$
\frac{\partial G_{\omega}}{\partial y_{i}}=E_{i} G_{\omega}, \text { avec }\left\{\begin{array}{l}
E_{1}=i \frac{K}{\beta^{2}}\left[M+\frac{x-x_{0}}{S_{0}}\left[1-i \frac{\beta^{2}}{K S_{0}}\right]\right] \\
E_{2}=i K \frac{y-y_{0}}{S_{0}}\left[1-i \frac{\beta^{2}}{K S_{0}}\right] \text { et } E_{3}=i K \frac{z-z_{0}}{S_{0}}\left[1-i \frac{\beta^{2}}{K_{0}}\right]
\end{array}\right.
$$

Supposons les sources sonores en rotation uniforme à la vitesse angulaire $\Omega=2 \pi \mathrm{N}$ autour de l'axe Ox, direction portant la vitesse d'écoulement $U$ (ceci exclut le cas où le rotor est mis en incidence). Si le rotor possède $\mathrm{B}$ aubes équidistantess, la période temporelle vaut $\mathrm{T}=1 /(\mathrm{BN})$. La surface $\mathrm{A}$ dans $(5)$ est alors relative à une seule aube et les fréquences acoustiques émises sont limitées aux harmoniques de la rotation de rang $n B$, puisque $\omega=n B \Omega$. Une source en $\vec{y}$ est aussi repérée par ses coordonnées cylindriques $r, \psi, x_{0}$ (fig. 1). $V_{n}$ est indépendante de $\psi$ et $f_{i}(\vec{y})$ peut être développée en série de Fourier :

$$
f_{i}=\sum_{s=-\infty}^{+\infty} F_{i}^{(s)} e^{i s \psi} \text { et } F_{i}^{(s)}=\frac{1}{2 \pi} \int_{0}^{2 \pi} f_{i} e^{-i s \psi} d \psi \quad(i=1,2,3)
$$

où $F_{i}^{(s)}$ est l'harmonique de charge de rang $s$, dépendant de la position sur la surface $A$ d'une aube mais non de $\psi$. Plus précisément, un point sur A est repéré à $\tau=0$ par ses coordonnées $\mathrm{r}, \psi_{\mathrm{c}}$ et $\mathrm{x}_{0}$. Les valeurs de $\psi_{\mathrm{c}}$, comprises entre $\psi_{1}(\mathrm{r})$ et $\psi_{2}(\mathrm{r})$, traduisent l'étendue angulaire de la projection transversale de la corde du profil à une envergure $r$; en pratique, tous ces angles sont voisins (autour de zéro par exemple). Ainsi :

$$
\psi=\Omega \tau+\psi_{\mathrm{c}}=2 \pi N \tau+\psi_{\mathrm{c}}
$$

et les grandeurs indépendantes de $\psi$ le sont aussi de $\tau$. 


\subsection{Approximation en champ lointain}

Les développements exposés jusqu'ici présentent l'intérêt sur les travaux déjà publiés d'être très généraux, valables même en champ proche. L'approximation de champ lointain consiste à supposer les distances d'observation grandes devant les dimensions de la source et les longueurs d'ondes $\lambda=2 \pi / \mathrm{K}$, soit :

$$
\frac{\mathrm{x}_{0}}{\mathrm{~S}_{0}} \ll 1, \frac{\mathrm{y}_{0}}{\mathrm{~S}_{0}} \ll 1, \frac{\mathrm{z}_{0}}{\mathrm{~S}_{0}} \ll 1 \text { et } \mathrm{KS}_{0} \gg 1
$$

Ainsi, $\alpha$ et les $\mathrm{E}_{\mathrm{i}}$ dans (7) et (8) sont indépendants de $\mathrm{y}_{0}$ et $\mathrm{z}_{0}$, c'est-à-dire de $\psi$ ou $\tau$. La relation (5) devient en tenant compte de (9) :

$$
P(\omega, \vec{x})=\int_{A} \rho_{0} V_{n} \alpha\left[\int_{\tau=0}^{T} G_{\omega} d \tau\right] d A+\sum_{s=-\infty}^{+\infty} \int_{A} F_{i}^{(s)} E_{i}\left[\int_{\tau=0}^{T} G_{\omega} e^{i s \psi} d \tau\right] d A
$$

Les intégrales sur le temps retardé sont calculées en développant $S_{0}$ et $\tau_{0}$ au premier ordre, l'ordre zéro étant :

$$
S=\sqrt{x^{2}+\beta^{2}\left(y^{2}+z^{2}\right)} \text { et } \sigma=\frac{M x+S}{\beta^{2}} \text { avec toujours } \beta^{2}=1-M^{2}
$$

Elles s'expriment en fonctions de Bessel de première espèce, $J$, après avoir introduit aussi les coordonnées cylindriques $\mathrm{d}, \varphi, \mathrm{x}$ du récepteur (fig. 1).

Les formules générales, programmées à l'ONERA, sont finalement les suivantes :

$$
\mathrm{P}(\mathrm{nB} \Omega, \overrightarrow{\mathrm{x}})=\mathrm{P}_{\mathrm{e}}(\mathrm{nB} \Omega, \overrightarrow{\mathrm{x}})+\mathrm{P}_{\mathrm{c}}(\mathrm{nB} \Omega, \overrightarrow{\mathrm{x}})
$$

avec, pour le bruit d'épaisseur,

$$
P_{e}=-i \rho_{0} \frac{B f}{2 \beta^{2} S}\left[1+M \frac{x}{S}\right] e^{-i K \sigma} e^{-i n B(\varphi-\pi / 2)} \int_{A} V_{n} e^{i\left(K A_{1}+n B \psi_{e}\right)} J_{n B}\left[K r \frac{d}{S}\right] d A
$$

et, pour le bruit de charge,

$$
\begin{gathered}
P_{c}=i \frac{B f}{2 a \beta^{2} S} e^{-i K \sigma} \sum_{s=-\infty}^{+\infty} e^{-i(n B-s)(\varphi-\pi / 2)} \int_{A}\left[\left[M+\frac{x}{S}\right] F_{x}^{(s)}+\beta^{2} \frac{y}{S} F_{y}^{(s)}+\beta^{2} \frac{z}{S} F_{z}^{(s)}\right] \\
\cdot e^{i\left(K A_{1}+n B \psi_{o}\right)} J_{n B-s}\left(K r \frac{d}{S}\right) d A
\end{gathered}
$$

où $A_{1}=\frac{x_{0}}{\beta^{2}}\left[M+\frac{x}{S}\right]$ exprime le fait que les aubes ne sont pas planes (elles ne sont pas contenues dans le plan de rotation $\mathrm{x}_{0}=0$ ). Il est rappelé que :

$$
K=\frac{2 \pi f}{a}=\frac{2 \pi n B N}{a} \text { donc } K r \frac{d}{S}=n B M_{r} \frac{d}{S}
$$

où $\mathrm{M}_{\mathrm{r}}=\Omega \mathrm{r} / \mathrm{a}$ est le nombre de Mach de rotation au rayon $\mathrm{r}$.

La transformation de Fourier $\mathrm{P}(\mathrm{nB} \Omega, \overrightarrow{\mathrm{x}})$ est calculée sur les fréquences positives et négatives : $\mathrm{n}$ varie de $-\infty$ à $+\infty$ dans (4). Le spectre acoustique, exprimé seulement sur les fréquences positives, est donc donné par :

$$
2|\mathrm{P}(\mathrm{n} B \Omega, \overrightarrow{\mathrm{x}})|^{2}=2\left|\mathrm{P}_{\mathrm{c}}(\mathrm{nB} \Omega, \overrightarrow{\mathrm{x}})+\mathrm{P}_{\mathrm{c}}(\mathrm{nB} \Omega, \overrightarrow{\mathrm{x}})\right|^{2} \quad(\mathrm{n}>0)
$$


Les relations (14) se simplifient dans certains cas. Si l'on néglige la vitesse d'écoulement $(M \simeq 0$ et $\beta^{2} \simeq 1$ ), il vient, en introduisant les coordonnées sphériques $D, \theta, \varphi$ de $\vec{x}$ (fig. 1) :

$$
\mathrm{s}=\sigma=\mathrm{D}, \mathrm{Kr} \frac{\mathrm{d}}{\mathrm{S}}=\mathrm{nBM}, \sin \theta, \mathrm{A}_{1}=\mathrm{x}_{0} \cos \theta
$$

Il s'ensuit en particulier que la composante $P \mathrm{e}^{\mathrm{i} \omega t}$ de la pression sonore (14) à la pulsation $\omega=\mathrm{n} B \Omega$ contient en facteur le terme (1/D) $\mathrm{e}^{i \omega(\mathrm{t}-\mathrm{D} / \mathrm{a})}$, caractéristique d'une onde sphérique. Le terme entre crochets de $\mathrm{P}_{c}$ dans (14) s'écrit simplement $: \overrightarrow{\mathrm{F}}^{(s)} \cdot \frac{\overrightarrow{\mathrm{x}}}{\mathrm{D}}$. Il est souvent commode de décomposer $\overrightarrow{\mathrm{F}}^{(s)}$ en termes de poussée $F_{T}^{(s)}$, axiale, et de traînée $F_{D}^{(s)}$, tangentielle. L'expression du bruit de charge $P_{c}$ est alors identique à celle de Lowson [4]. Si, de plus, le rotor est isolé, seule subsiste la charge moyenne $(s=0)$ et $P_{c}$ se réduit au résultat que Gutin a établi pour une hélice dès 1936 [5].

\section{ACOUSTIQUE DES ROTORS CARENES}

\subsection{Formules en conduit cylindrique infiniment long}

L'espace est limité ici à un conduit cylindrique de rayon $R$, infiniment long et à parois parfaitement rigides. Rotor et récepteur sont donc situés à l'intérieur de ce conduit. La démarche adoptée par Goldstein en [6] ou [1, chapitre 4] part toujours de la relation (1) ; la fonction de Green en espace libre convecté est remplacée par celle en cylindrique satisfaisant à la condition à la limite du conduit (dérivée radiale nulle au rayon $\mathrm{R}$ ). Ainsi :

$$
\mathrm{G}(\overrightarrow{\mathrm{y}}, \tau ; \overrightarrow{\mathrm{x}}, \mathrm{t})=-\frac{\mathrm{i}}{2} \sum_{\mathrm{m}=-\infty}^{+\infty} \sum_{\mu=1}^{+\infty} \frac{\mathrm{J}_{\mathrm{m}}\left(\mathrm{k}_{\mathrm{T}} \mathrm{r}\right) \mathrm{J}_{\mathrm{m}}\left(\mathrm{k}_{\mathrm{T}} \mathrm{d}\right)}{\Gamma} \cdot \frac{\mathrm{e}^{\mathrm{j} \mathrm{m}(\psi-\varphi)}}{\Delta} \cdot \delta\left(\mathrm{t}-\tau-\frac{\mathrm{k}}{\mathrm{K}} \cdot \frac{\mathrm{x}-\mathrm{x}_{0}}{\mathrm{a}}\right)
$$

où $\mathrm{K}, \mathrm{k}_{\mathrm{T}}$ et $\mathrm{k}$ sont les nombres d'ondes, respectivement total, transversal et longitudinal,

$$
\begin{aligned}
& \Delta=\left(\mathrm{K}^{2}-\left(1-\mathrm{M}^{2}\right) \mathrm{k}_{\mathrm{T}}^{2}\right]^{1 / 2} \text { sera interprété au paragraphe suivant, } \\
& \Gamma=\pi\left[\mathrm{R}^{2}-\frac{\mathrm{m}^{2}}{\mathrm{k}_{\mathrm{T}}^{2}}\right] \mathrm{J}_{\mathrm{m}}^{2}\left(\mathrm{k}_{\mathrm{T}} \mathrm{R}\right) \text { est un facteur de normalisation. }
\end{aligned}
$$

Le reste des calculs suit à peu près les mêmes étapes qu'au paragraphe 2, à l'exception près qu'il n'existe pas d'approximation en champ lointain. La charge $\vec{F}^{(s)}$ est décomposée en poussée $F_{T}^{(s)}$ et traînée $F_{D}^{(s)}$, en négligeant la force radiale. Dans ces conditions, les termes sources suivants apparaissent :

source associée à l'épaisseur, $\mathrm{E}_{\mathrm{m} \mu}=\int_{\mathrm{A}} \mathrm{V}_{\mathrm{n}} \mathrm{J}_{\mathrm{m}}\left(\mathrm{k}_{\mathrm{T}} \mathrm{r}\right) \mathrm{e}^{\mathrm{i}\left(\mathrm{nB} \psi_{\mathrm{c}}+\mathrm{kx} \mathrm{x}_{\mathrm{o}}\right)} \mathrm{dA}$
sources dues à la poussée, $\mathrm{T}_{\mathrm{m} \mu}^{(\mathrm{s})}=\int_{\mathrm{A}} \mathrm{F}_{\mathrm{T}}^{(\mathrm{s})} \mathrm{J}_{\mathrm{m}}\left(\mathrm{k}_{\mathrm{T}} \mathrm{r}\right) \mathrm{e}^{\mathrm{i}\left(\mathrm{nB} \psi_{\mathrm{c}}+\mathrm{kx}_{\mathrm{o}}\right)} \mathrm{dA}$
sources dues à la traînée, $\mathrm{D}_{\mathrm{m} \mu}^{(\mathrm{s})}=\int_{\mathrm{A}} \frac{1}{\mathrm{r}} \mathrm{F}_{\mathrm{D}}^{(\mathrm{s})} \mathrm{J}_{\mathrm{m}}\left(\mathrm{k}_{\mathrm{T}} \mathrm{r}\right) \mathrm{e}^{\mathrm{i}\left(\mathrm{nB} \psi_{\mathrm{c}}+\mathrm{kx} \mathrm{x}_{\mathrm{o}}\right)} \mathrm{dA}$


Il faut noter que $T_{m \mu}^{(s)}$ a la dimension d'une force, tandis que $D_{m \mu}^{(s)}$ a celle d'une force par unité de longueur. Finalement, (14) est remplacé par :

$$
\mathrm{P}(\mathrm{nB} \Omega, \overrightarrow{\mathrm{x}})=\mathrm{P}_{\mathrm{e}}(\mathrm{nB} \Omega, \overrightarrow{\mathrm{x}})+\mathrm{P}_{\mathrm{c}}(\mathrm{nB} \Omega, \overrightarrow{\mathrm{x}})
$$

avec, pour le bruit d'épaisseur,

$$
P_{e}=-\frac{1}{2} \rho_{0} a B \sum_{\mu=1}^{+\infty} \frac{K-M k}{\Gamma \Delta} E_{n B, \mu} J_{n B}\left(k_{T} d\right) e^{-i(n B \varphi+k x)}
$$

et, pour le bruit de charge,

$$
\mathrm{P}_{\mathrm{c}}=\frac{1}{2} \mathrm{~B} \sum_{\mu=1}^{+\infty} \sum_{s=-\infty}^{+\infty} \frac{1}{\Gamma \Delta}\left[k \mathrm{~T}_{m \mu}^{(s)}+m D_{m \mu}^{(s)}\right] \mathrm{J}_{m}\left(k_{\mathrm{T}} \mathrm{d}\right) \mathrm{e}^{-\mathrm{i}(m \varphi+k x)} \text {, où } \mathrm{m}=\mathrm{nB}-\mathrm{s}
$$

Il ressort en particulier que la composante $\mathrm{Pe}^{\mathrm{i} \omega t}$ de la pression sonore à la pulsation $\omega=\mathrm{nB} \Omega$ en un point de réception $\bar{x}(d, \varphi, x)$ est la somme de termes :

$$
\text { C } J_{m}\left(k_{T} d\right) e^{i(\omega t-m \varphi-k x)}
$$

Ils représentent les ondes hélicoïdales (ou modes tournants) dans le conduit (avec $\mathrm{m}=\mathrm{nB}$ pour le bruit d'épaisseur). Le champ acoustique est donc décomposé sur les fonctions propres de l'équation d'onde en géométrie cylindrique; $\mathrm{m}$ est le nombre d'ondes angulaire et $\mu$ le mode radial.

Les résultats précédents fournissent le champ sonore à l'intérieur d'un conduit infini. Le rayonnement en champ libre peut être obtenu en le coupant en $\mathrm{x}=\mathrm{L}$ et en supposant que les réflexions sur l'ouverture sont suffisamment faibles pour que la solution (20) reste valable. Le calcul le plus simple est fourni par Tyler et Sofrin, qui considèrent que le champ acoustique incident est équivalent à un ensemble de monopôles dans la section de sortie, possédant des relations de phase entre eux [7]. Ceci est une généralisation du problème classique du rayonnement d'un piston plan bafflé. Une onde élémentaire (21) donne finalement en champ lointain, en un point de coordonnées sphériques $(D, \theta, \phi)$ repérées maintenant par rapport au centre de la section de sortie et non plus du rotor :

$$
p(D, \theta, \phi, t)=i^{m+1} \frac{C k R^{2}}{2 D} e^{i \omega(t-D / a)} e^{-i(m \phi+k L)} J_{m}\left(k_{T} R\right)\left[\frac{2 K R \sin \theta}{\left(k_{T} R\right)^{2}-(K R \sin \theta)^{2}} \cdot J_{m}^{\prime}(K R \sin \theta)\right]
$$

où $\mathrm{J}_{\mathrm{m}}^{\prime}$ est la dérivée de $\mathrm{J}_{\mathrm{m}}$. L'angle $\theta$ est limité à $[0, \pi / 2]$ à cause de l'hypothèse d'encastrement dans un plan parfaitement rigide. Le terme de (22) entre crochets détermine la directivité.

\subsection{Propriétés de coupure du conduit}

Les phénomènes de coupure jouent un rôle fondamental dans l'émission sonore d'un rotor caréné. Ils se déduisent de la relation de dispersion entre les nombres d'ondes $K, k_{T}$ et $k$ introduits au paragraphe précédent :

$$
(\mathrm{K}-\mathrm{Mk})^{2}=\mathrm{k}^{2}+\mathrm{k}_{\mathrm{T}}^{2}
$$

C'est une équation du second degré en $\mathrm{k}$, dont le discriminant réduit $\Delta^{2}$, écrit après la relation (18), doit être positif pour que $k$ soit réel et que l'onde (21) se propage. 
Si le conduit est parfaitement rigide, la condition d'annulation de la vitesse vibratoire en paroi donne :

$$
\mathrm{J}_{\mathrm{m}}^{\prime}\left(\mathrm{k}_{\mathrm{T}} \mathrm{R}\right)=0 \text {, soit } \mathrm{k}_{\mathrm{T}}=\chi_{\mathrm{m} \mu} / \mathrm{R}
$$

où $\chi_{\mathrm{m} \mu}$ est l'abscisse du $\mu^{\text {ème }}$ zéro de la dérivée $\mathrm{J}_{\mathrm{m}}^{\prime}$. Ceci montre que $\mathrm{k}_{\mathrm{T}}$, et donc $\mathrm{k}, \Delta, \Gamma$, dépendent de $\mathrm{m}$ et $\mu$, même si ces indices sont omis dans ce qui précède pour alléger les notations. La condition $\Delta^{2} \geq 0$ implique :

$$
K \geq \sqrt{1-M^{2}} k_{T} \text { ou }|f| \geq f \text { avec } f_{c}=\sqrt{1-M^{2}} \frac{a \chi_{m \mu}}{2 \pi R}
$$

$\mathrm{f}_{\mathrm{c}}$ étant la fréquence de coupure du mode acoustique $(\mathrm{m}, \mu)$.

Bruit d'épaisseur et de charge moyenne. Ces deux composantes sont réunies par le fait que $\mathrm{s}=0$ ou $\mathrm{m}=\mathrm{nB}$, rang de l'harmonique de la rotation considéré, dans les expressions (20). Examinons ce qu'implique la propagation dans le conduit. Comme $\mathrm{f}=\mathrm{nBN}$ et que $\chi_{\mathrm{m} \mu}>\chi_{\mathrm{m} 1}>|\mathrm{m}|$ mais que $\chi_{\mathrm{m} 1}$ est peu supérieur à $|\mathrm{m}|$, une condition nécessaire (mais non strictement suffisante) est, par (25) :

$$
\begin{aligned}
& |\mathrm{n}| \mathrm{BN} \succ \sqrt{1-\mathrm{M}^{2}} \frac{\mathrm{a}|\mathrm{m}|}{2 \pi \mathrm{R}} \text {, soit, puisque } \mathrm{m}=\mathrm{nB}, \\
& \frac{2 \pi \mathrm{RN}}{\mathrm{a}} \succ \sqrt{1-\mathrm{M}^{2}} \text { ou } \mathrm{M}_{\mathrm{R}} \succ \sqrt{1-\mathrm{M}^{2}} \text { ou } \underline{\mathrm{M}_{\text {rel }}} \succ 1
\end{aligned}
$$

$M_{\text {rel }}=\sqrt{M_{R}^{2}+M^{2}}$ étant le nombre de Mach relatif en bout d'aube. Ainsi, les termes étudiés ne rayonnent en champ libre que pour des rotors transsoniques (en supposant que la manche est suffisamment longue). Ils constituent alors la principale source sonore.

Bruit d'interaction. Le bruit de raie d'un rotor subsonique est done seulement dû aux interactions entre rotor et stator ou distorsion d'écoulement (harmoniques de charge de rang $\mathrm{s} \neq 0$ ). Comme $\mathrm{m}=\mathrm{nB}-\mathrm{s}$ d'après (20), l'inégalité (26) devient :

$$
\frac{|\mathrm{n}| \mathrm{BM}_{\mathrm{R}}}{\sqrt{1-\mathrm{M}^{2}}} \succ|\mathrm{nB}-\mathrm{s}| \text { d'où }\left[1-\frac{\mathrm{M}_{\mathrm{R}}}{\sqrt{1-\mathrm{M}^{2}}}\right)|\mathrm{n}| \mathrm{B} \prec|\mathrm{s}| \prec\left[1+\frac{\mathrm{M}_{\mathrm{R}}}{\sqrt{1-\mathrm{M}^{2}}}\right]|\mathrm{n}| \mathrm{B}(28)
$$

Ceci définit la plage d'harmoniques de charge gênants, centrée sur la composante de rang $|\mathrm{n}| \mathrm{B}$ :

$$
0<s_{\min }<|s|<s_{\max } \text {. }
$$

\section{QUELQUES EXEMPLES D'APPLICATION AUX COMPRESSEURS}

\subsection{Intérêt des calculs sur rotor libre pour les compresseurs}

On peut se demander quelle valeur peut être accordée à des calculs acoustiques de compresseur ne prenant pas en compte la présence du conduit. Tout d'abord, Lowson a remarqué que les termes dominants dans (14) et (20) sont les mêmes [4]. Pour un rotor subsonique, l'argument est fondé sur l'allure des fonctions de Bessel, dont l'amplitude devient notable seulement quand, en gros, l'argument est supérieur à l'ordre (en module), soit dans (14) simplifié par (17) :

$$
|n| B M_{r} \sin \theta \succ|n B-s| \text { ou a fortiori }|n| B M_{r} \succ|n B-s|
$$

ce qui est exactement (28) à faible vitesse d'avancement $\left(\sqrt{1-M^{2}} \simeq 1\right)$. Ceci n'implique cependant pas que la présence d'un conduit ne change pas le rayonnement en champ libre. 
Quant aux rotors transsoniques, il a été trouvé que les termes dominants sont ceux d'épaisseur et de charge moyenne, c'est-à-dire rigoureusement les mêmes que pour une hélice libre [10]. En outre, Eversman a récemment montré par des calculs numériques que les niveaux sonores et les directivités sont peu modifiés, qu'il existe ou non un carénage autour du rotor [9].

\subsection{Etude des soufflantes transsoniques}

L'étude des rotors transsoniques présente de l'intérêt en particulier dans le domaine aéronautique car la soufflante des turboréacteurs modernes est souvent transsonique et pourrait être encore plus rapide dans la prochaine génération de moteurs.

Même si l'on suppose le rotor libre $(\S 2)$, il faut insister sur le fait que les directivités à grande vitesse sont assez différentes de celles d'une hélice subsonique classique. Considérons une source en rotation à un rayon efficace $r$ et négligeons la vitesse d'avancement $(M=0)$, pour simplifier au maximum. La directivité du bruit d'épaisseur, par exemple, est déterminée à une fréquence $\mathrm{f}$ par le facteur suivant de la valeur efficace de $\mathrm{P}_{\mathrm{e}}$ :

$$
\mathrm{Kr} \mathrm{J}_{\mathrm{nB}}(\mathrm{Kr} \sin \theta)=\mathrm{nBM}_{\mathrm{r}} \mathrm{J}_{\mathrm{nB}}\left(\mathrm{nBM}_{\mathrm{r}} \sin \theta\right)
$$

Son logarithme est porté en fonction de $\theta$ sur la figure 2, pour $n B=22$ et avec $M_{r}$ en paramètre. Tant que $M_{r}$ est subsonique, le maximum est situé dans le plan de l'hélice $\left(\theta=90^{\circ}\right)$ et croît fortement avec $M_{r}$ (écart de $42 \mathrm{~dB}$ entre $M_{r}=0,7$ et $M_{r}=1$ ). Ensuite, il n'augmente plus qu'en $20 \log M_{r}$ quand $J_{n B}\left(\chi_{n B, 1}\right)$ peut être atteint, soit, pour $n B=22, \chi_{n B, 1}=24,29$ et $M_{r} \succ 1,1$. Les maxima successifs des courbes sont tels que $J_{\mathrm{nB}}^{\prime}(\mathrm{Kr} \sin \theta)=0$, soit, compte tenu de la définition des $\chi_{\mathrm{m} \mu}$ en (24):

$$
\sin \theta_{\max }=\frac{\chi_{\mathrm{m} \mu}}{\mathrm{Kr}}=\frac{\chi_{\mathrm{m} \mu}}{\mathrm{nBM}_{\mathrm{r}}} \text {, avec } \mathrm{m}=\mathrm{nB} \text { et } \mu=1,2,3, \cdots
$$

jusqu'à ce que le second membre soit supérieur à 1 , auquel cas $\theta_{\max }=90^{\circ}$. Les lobes, associés aux oscillations de la fonction de Bessel, sont donc de plus en plus nombreux et les maxima de plus en plus axiaux quand $M_{r}$ augmente. En réalité, $M_{r}$ est proportionnel à $r$ et l'intégration en envergure aboutit à une courbe lissée, avec un plateau qui s'élargit davantage aux grandes valeurs du nombre de Mach périphérique.

Ces résultats sont obtenus sans prendre en compte le carénage. Cependant, les mêmes tendances se retrouvent pour le rayonnement par l'embouchure d'un conduit cylindrique : la figure 3 est calculé par la formule (22), toujours pour $\mathrm{m}=\mathrm{nB}=22$ et $\mu=1$, en prenant $\mathrm{D} / \mathrm{R}=1$ (bien que l'expression ne soit valable qu'en champ lointain) et un niveau de pression sonore de $0 \mathrm{~dB}$ en paroi du conduit: $20 \log \left|C J_{m}\left(k_{T} R\right)\right|=0 \mathrm{~dB}$. Il est connu que la directivité dépend essentiellement du rapport de coupure [11]:

$$
\xi=\frac{f_{c}}{f}=\frac{k_{T}}{K}=\sqrt{1-M^{2}} \frac{\chi_{m \mu}}{K R}=\frac{\chi_{m \mu}}{K R} \quad(0<\xi<1 \text { pour un mode propagatif }) .
$$

Rice a montré en particulier que la direction du maximum est donnée approximativement par [12] :

$$
\sin \theta_{\max }=\xi
$$

ce qui est rigoureusement l'expression (29). Ceci se retrouve d'ailleurs aisément par un tracé de rayons (fig. 4). Le tableau I réunit les valeurs $\theta_{\max }$ du premier maximum $(\mu=1)$, qui est le maximum maximorum. La colonne relative au rotor libre est déduite de la relation (29) et correspond évidemment à la figure 2. La colonne du rotor caréné est issue de la figure 3 (les angles sont arrondis à l'entier le plus proche). Comme il fallait s'y attendre, les angles ne sont pas identiques dans les deux cas ; ils restent cependant voisins et évoluent de la même manière. 
Tableau I - Direction du rayonnement maximum $(m=n B=22)$.

\section{CONCLUSION}

\begin{tabular}{|c|c|c|}
\hline \multirow{2}{*}{$M_{\mathrm{R}}$} & \multicolumn{2}{|c|}{$\theta_{\max }$ (degrés) } \\
\cline { 2 - 3 } & Rotor libre & Rotor caréné \\
\hline \hline 1,5 & 47,4 & 52 \\
1,37 & 53,7 & 59 \\
1,25 & 62,0 & 70 \\
1,2 & 66,9 & 78 \\
1,104 & 90 & 90 \\
$<1,1$ & 90 & coupé \\
\hline
\end{tabular}

Ce document a tenté de montrer que des modèles théoriques sont disponibles pour prévoir le bruit de raie émis par un rotor. L'application des formules s'est limitée dans ce qui précède à quelques discussions sur les rotors transsoniques. C'est la situation la plus simple pour les calculs car les sources sonores principales sont dues à l'épaisseur des aubes et à leur charge moyenne. En outre, la présence d'un carénage ne modifie pas fondamentalement le rayonnement en champ libre. Il reste à répondre à une question importante, celle de la contribution relative du terme quadripolaire (lié aux ondes de Mach) sur les harmoniques de la fréquence de passage des aubes, composante qui a été négligée dans tout ce travail. Le cas des compresseurs subsoniques est plus complexe. En effet, seuls des harmoniques de charge de rang relativement élevé produisent des ondes acoustiques à structure spatiale suffisamment simple pour qu'elles se propagent dans le conduit entourant le rotor. La principale difficulté provient des données aérodynamiques car les programmes de calcul ne sont pas encore capables de prévoir ces fluctuations de charge en haute fréquence. L'effort doit de toute évidence porter sur ce sujet.

Un autre problème se pose en pratique. Il a été affirmé en introduction que les méthodes semi-empiriques ne sont pas assez fiables pour être employées au stade des avant-projets et que des modèles purement théoriques sont indispensables. Toutefois, il n'est guère envisageable de mener un calcul aérodynamique (et donc acoustique) précis lors de cette étape pour définir la machine, à cause de l'absence de certaines données et des temps d'ordinateur. Ceci constitue un thème de réflexion pour l'avenir : il faudra rechercher comment mener une analyse paramétrique, un peu comme ce qui a été ébauché dans la dernière partie de cette communication, afin d'aboutir in fine à une optimisation aéroacoustique.

\section{REMERCIEMENTS}

Ces études sont supportées par des contrats avec la SNECMA.

\section{REFERENCES}

[1] Goldstein M.E., Aeroacoustics (McGraw-Hill International Book Company, New York, 1976). [2] Ffowes Williams J.E. and Hawkings D.L., Philosophical Transactions of the Royal Society of London, Vol. 264, Series A, No. 1151 (mai 1969) 321-342.

[3] Hanson D.B., AIAA Journal, 18, No. 10 (octobre 1980) 1213-1220.

[4] Lowson M.V., J. Acoust. Soc. Am., 47, No. 1, Part 2 (janvier 1970) 371-385.

[5] Gutin L. Ya., "On the sound field of a rotating propeller", Physikalische Zeitschrift der Sowjetunion, 9, No. 1 (1936) 57-71 (en russe) \& NACA TM-1195, Washington, D.C. (1948).

[6] Goldstein M., J. Acoust. Soc. Am., 56, No. 2 (août 1974) 497-509. Tyler J.M. and Sofrin T.G., Society of Automotive Engineers Transactions, 70 (1962) 309-332. Schulten J.B.H.M., "Sound generation by ducted fans and propellers as a lifting surface problem", Thèse, CIP-Data Koninklijke Bibliotheek, La Haye, Pays-Bas (18 février 1993).

[ 9] Eversman W., "Comparison of radiated noise from shrouded and unshrouded propellers", Second International Congress on Recent Developments in Air- and Structure-Borne Sound and Vibration, Auburn University, USA (4-6 mars 1992) 121-128.

[10] Gounet H. and Léwy S., Journal of Aircraft, 25, No. 5 (mai 1988) 428-435.

[11] Léwy S., "Exact and simplified computation of noise radiation by an annular duct", Internoise 88, Avignon (30 août-1 ${ }^{\text {er }}$ septembre 1988), Proceedings Vol. 3, 1559-1564.

[12] Rice E.J. AIAA Journal, 16, No. 9 (septembre 1978) 906-911. 


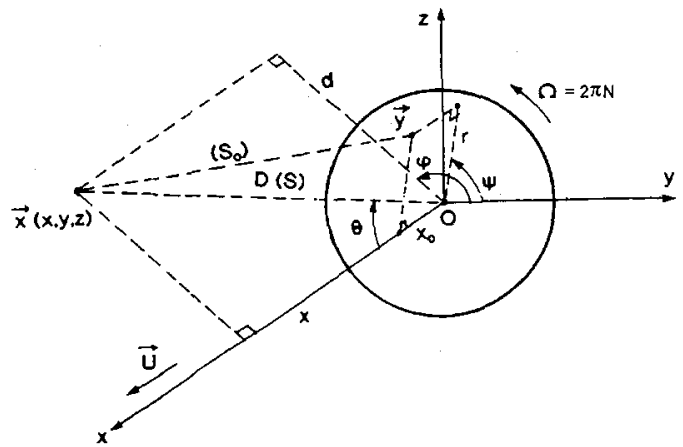

\begin{tabular}{|c|c|c|c|}
\hline Coordonnées & cartésiennes & cylindriques & sphériques \\
\hline \hline Source $\overrightarrow{\mathrm{y}}$ & $\begin{array}{c}\mathrm{y}_{1}, \mathrm{y}_{2}, \mathrm{y}_{3} \\
\text { ou } \\
\mathrm{x}_{0}, \mathrm{y}_{0}, \mathrm{z}_{0}\end{array}$ & $\mathrm{r}, \psi, \mathrm{x}_{0}$ & - \\
\hline Récepteur $\overrightarrow{\mathrm{x}}$ & $\mathrm{x}, \mathrm{y}, \mathrm{z}$ & $\mathrm{d}, \varphi, \mathrm{x}$ & $\mathrm{D}, \theta, \varphi(\mathrm{ou} \phi$ \\
\hline
\end{tabular}

Figure 1

Définition de la géométrie et des coordonnées utilisées.

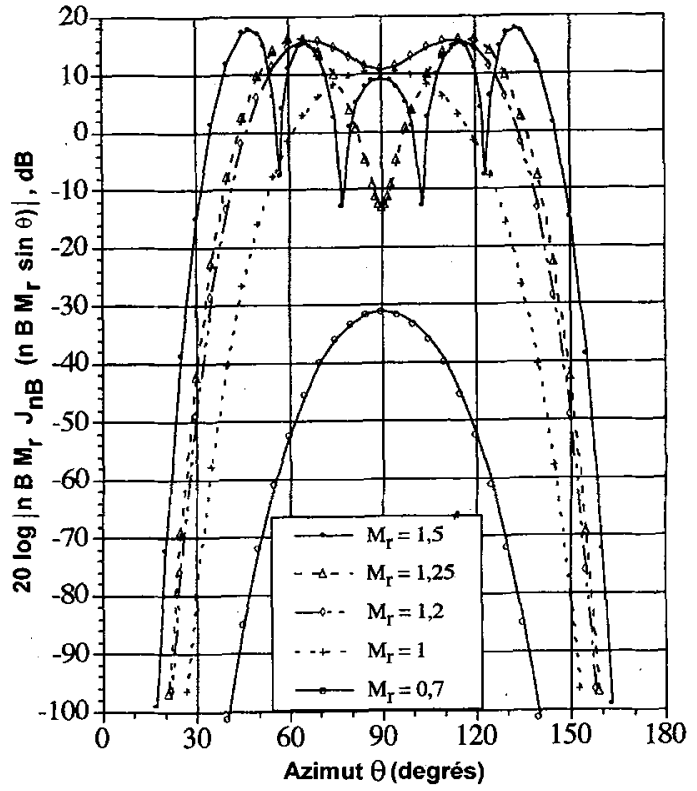

Figure 2

Effet du nombre de Mach de rotation

sur la directivité du bruit d'épaisseur engendré par un rotor libre : $\mathrm{nB}=22$. 


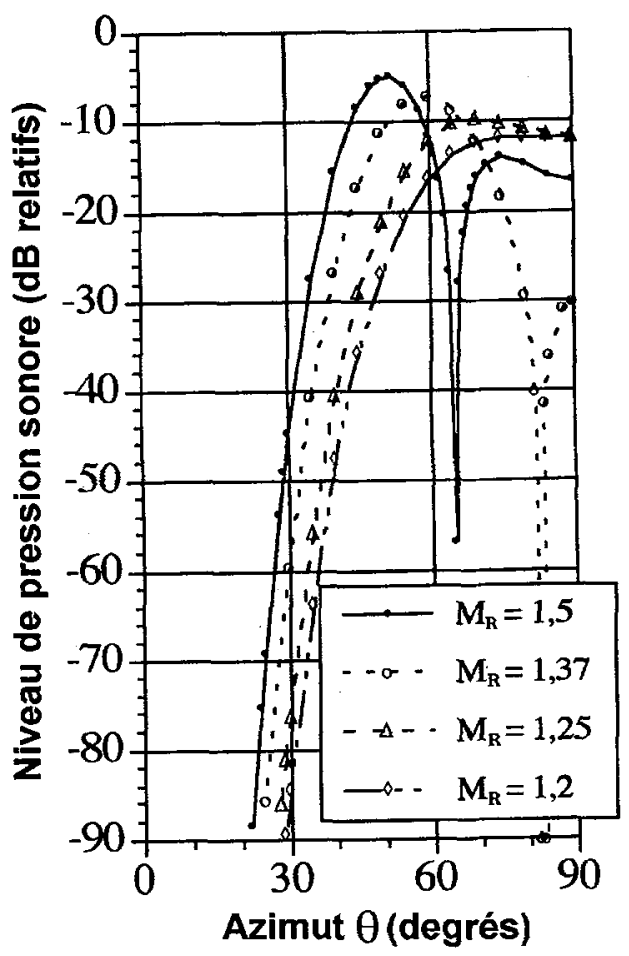

Figure 3

Effet du nombre de Mach de rotation

sur la directivité rayonnée par un rotor transsonique caréné : $m=n B=22, \mu=1$.

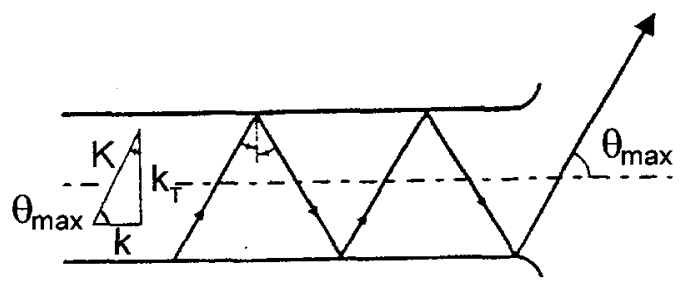

Figure 4

Tracé de rayons, schématisant la propagation acoustique dans un conduit et le rayonnement en champ libre. 